\title{
Use of fractal models to define the scaling behavior of the aquifers' parameters at the mesoscale
}

\author{
C. Fallico $^{1}$ - S. De Bartolo ${ }^{2}$ G. F. A. Brunetti ${ }^{1}$ G. Severino ${ }^{3}$ (b)
}

Accepted: 19 September 2020/Published online: 3 October 2020

(C) The Author(s) 2020

\begin{abstract}
We present an experimental study aiming at the identification of the hydraulic conductivity in an aquifer which was packed according to four different configurations. The conductivity was estimated by means of slug tests, whereas the other parameters were determined by the grain size analysis. Prior to the fractal we considered the dependence of the conductivity upon the porosity through a power (scaling) law which was found in a very good agreement within the range from the laboratory to the meso-scale. The dependence of the conductivity through the porosity was investigated by identifying the proper fractal model. Results obtained provide valuable indications about the behavior, among the others, of the tortuosity, a parameter playing a crucial role in the dispersion phenomena taking place in the aquifers.
\end{abstract}

Keywords Fractal models $\cdot$ Scaling behavior $\cdot$ Hydraulic conductivity $\cdot$ Porosity $\cdot$ Tortuosity

\section{Introduction}

The study of flow and transport in groundwater is affected by uncertainty, mainly addressed to the impossibility to get a direct and timely access to the porous medium. As a consequence, the aquifers' characterization is commonly faced by considering average values of the various parameters, determined by laboratory and/or field measurements. Such an uncertainty is then propagated in the models of flow and transport processes (Severino et al. 2009; Fallico et al. 2010). A way to copy with this increasing lack of knowledge in the field and regional scale models is related to the representative elementary volume that increases with the characteristic length of the medium (Winter and Tartakovsky 2001; Fallico et al. 2012; Severino and Coppola 2012). This led to the development of correlation models between aquifer volumes and related

G. Severino

severino@unina.it

1 Department of Civil Engineering, University of Calabria, Rende, Italy

2 Department of Engineering for Innovation, University of Salento, Lecce, Italy

3 Department of Agricultural Sciences, University of NaplesFederico II, Naples, Italy characteristic parameters (Hyun et al. 2002; Severino et al. 2006). There are several studies (see, e.g. Severino et al. 2019, Fallico et al. 2018) about scaling laws with more general validity, but as the level of generalization increases there is inevitably an increase of the uncertainty affecting the result obtained (Severino 2011a; Severino et al. 2011). In any case, the development of studies on scaling laws has stimulated investigations on some aspects of huge importance, such as the identification of the mechanisms influencing the scaling behavior, and the identification of the particular scale of investigation (see, e.g. Di Federico et al. 1999; Severino and Santini 2005). Some of this crucial topics are addressed in detail, later on in the present study.

Among the parameters characterizing the aquifers, hydraulic conductivity is certainly the most important for the description of water flow and mass transport phenomena in porous media. Studies showing a scaling behavior of hydraulic conductivity are numerous (Clauser 1992; Guimerà et al. 1995; Rovey and Cherkauer 1994; SchulzeMakuch and Cherkauer 1997, 1998; Schulze-Makuch et al. 1999; Fallico et al. 2010, 2012, 2016; Fallico 2014). Most of these studies show that hydraulic conductivity tends to increase when the scale increases and the causes of this behavior are commonly attributed to the medium heterogeneity. At the smaller scales, traditionally defined of laboratory, with the characteristic dimensions of the soil 
samples (between few centimeters up to $40-50 \mathrm{~cm}$ ), heterogeneity manifests its influence mainly through the shape and size of the pores and canaliculi, while on the major scales, traditionally defined of field, the influence of heterogeneity is mainly due to the tortuosity and the interconnection of the flow paths and canaliculi (Bouma 1982; Yanuka et al. 1986; Giménez et al. 1999; Knudby and Carrera 2006; Severino 2011b; Ghanbarian et al. 2012). However, the study of the intermediate scale between laboratory and field, characterized by dimensions ranging from a few tens of centimeters to a few meters, is becoming increasingly important. In fact, at this scale it is easier to study particular local phenomena, which often influence the flow and mass transport at larger scales with not negligible uncertainty (Comegna et al. 2010; Severino et al. 2010, 2012; Fallico et al. 2018). At this intermediate scale, the structural characteristics of the porous medium assume substantial importance, determining the heterogeneity that underlies the scaling behavior in question, namely porosity and tortuosity. Specifically, tortuosity, which characterizes the microstructure of the porous medium and through which it is possible to characterize the passage from laboratory to field scale, assumes great importance.

Therefore, this intermediate scale acts as a link between the laboratory scale and the field one, coexisting in it both ways in which the influence of heterogeneity occurs in the aforementioned two extreme scales and the passage from one to other. These concepts continue to be valid even for porosity, despite its scalar behavior presents still uncertainties, this is often taken as scale parameter to which correlate the hydraulic conductivity (Ahuja et al. 1989; Franzmeier 1991; Ewing et al. 2010; Fallico 2014; Agboola et al. 2017; Chen et al. 2020).

Numerous models have been developed to describe these behaviors using fractal geometry, starting from the studies of Jacquin and Adler (1987) and Muller and McCauley (1992), up to those of Xu and Yu (2008), De Bartolo et al. (2013), Chen and Yao (2017) and Chen et al. (2020).

The knowledge of the uncertainty with which the various models used in this context describe the phenomena in question is of fundamental importance. This is also and above all true from the practical and applicative point of view, taking into account, for example, that many situations concerning contaminated aquifers fall precisely into the intermediate scale considered here.

The main aim of this study is to provide useful information about the reliability of the main models used for the description of the phenomena in question, determining the uncertainty of the related parameters. Furthermore, among the aims of this study, it is necessary to include the verification of the scaling behavior of the hydraulic conductivity at the particular mesoscale taken into consideration, assuming porosity as scale parameter, verifying the influence that tortuosity, as heterogeneity manifestation of the porous medium, exerts on the phenomenon, bearing in mind that the latter parameter depends both on the measurement scale and on the fractal size of the tortuous capillaries (Wheatcraft and Tyler 1988; Vidales and Miranda 1996; Ewing et al. 2010).

Moreover, the results obtained by applying the fractal analysis to the experimental data considered here allow to characterize the behavior of the parameters under examination in the investigated mesoscale and for the porous media investigated, namely coarse grained aquifers, consisting mainly of sand, which are a type widely affected by application purposes. Therefore, the validity of the results obtained relates exclusively to the aforementioned areas of investigation.

Recalled the main topics debated in this study, the device built in laboratory to simulate a confined aquifer, on which the experimental part of the present study was carried out, is described. Afterwards, the methodologies considered for the measurement of parameters under examination and for the determination of the scaling laws, experimentally and by fractal geometry, are briefly recalled. Later, the results obtained are adequately shown and discussed. Finally, the main results obtained here are summarized.

\section{Materials}

\subsection{Experimental set-up}

The experimental device used in the present study was built in the Laboratory of Hydraulics of the Department of Civil Engineering of the University of Calabria (Italy). An accurate description of the device in question is reported in numerous studies in the literature (Fallico et al. 2018, 2020; Aristodemo et al. 2018), therefore only the main construction features of the device are mentioned here. The artificial aquifer was realized inside a steel box, with square base, a side equal to $2 \mathrm{~m}$ and $1 \mathrm{~m}$ height, with suitable lateral stiffeners. Inside the box, at distance of $5 \mathrm{~cm}$ from the walls of the box and along the whole perimeter, some vertical metallic supports were fixed, to which a metal mesh was anchored, on which a geotextile layer was disposed. Within the metal mesh the porous medium constituting the aquifer was placed, and between the metal mesh and the box wall a perimetric chamber was built in which the water can to flow, allowing to verify the hydraulic heads, fixed by two external loading reservoirs connected with the perimetric chamber, and compliance with the boundary conditions. Fixed the aquifer thickness, 
for the roof of this PVC panels of $2 \mathrm{~mm}$ of thickness were used, flexing them upwardly at the walls, so as to create a lateral coating, high about $25 \mathrm{~cm}$. To ensure stability of PVC panels, a second layer of porous medium was placed above. Inside the metal box, No. 10 fully penetrating wells were placed, spirally arranged, with a distance $(d)$ from the central well No. 1 gradually increasing of $10 \mathrm{~cm}$ away from the previous, along directions increased of $45^{\circ}$ from the previous one. Only the well No. 10 was placed along a direction increased by further $45^{\circ}$ so as not to place it too close to the metallic mesh. Each well shows a screened part corresponding to the entire thickness of the confined aquifer and an inner radius of $1.4 \mathrm{~cm}$. Figure 1 shows a planimetric and sectional representation of the metal box and the aquifer, with the layout of the wellsand the experimental device with the pressure transducers inserted in the wells.

The measurement of the hydraulic conductivity was carried out by slug test, injecting a fixed volume of water into the central well (No. 1) and acquiring the hydraulic head values by particular pressure transducers both in the injection well and in the other wells. To perform the slug tests, the following injection volumes were used: $0.30 \mathrm{~L}$, $0.40 \mathrm{~L}, 0.60 \mathrm{~L}, 0.70 \mathrm{~L}, 0.80 \mathrm{~L}, 0.90 \mathrm{~L}$. The slug tests were repeated in successive stages, for four different configurations of the porous medium making up the aquifer, using the same injection volumes. The aquifer thickness and the value of the undisturbed hydraulic head, characterizing the initial condition for each test, are shown in Table 1 for each of the four configurations considered.

\subsection{Characterization of the porous media}

The porous media used to build the confined aquifer in question were subjected to a careful particle size analysis,

Fig. 1 a Planimetric scheme of the metal box, with the layout of the wells. b Stratigraphic scheme. c Experimental device
Table 1 Formation thickness and undisturbed hydraulic heads for each configuration

\begin{tabular}{lllll}
\hline Configurations type & I & II & III & IV \\
\hline Formation thickness $t_{s}(\mathrm{~m})$ & 0.25 & 0.25 & 0.25 & 0.22 \\
Undisturbed hydraulic heads $(\mathrm{m})$ & 0.38 & 0.32 & 0.32 & 0.35 \\
\hline
\end{tabular}

determining their main granulometric and textural characteristics. Furthermore, porosity and effective porosity were measured in laboratory for each configuration, using the densimetric method. Specifically the porosity was determined by the following relationship (Lambe 1951; Danielson and Sutherland 1986):

$\phi=1-\frac{\rho_{\text {bulk }}}{\rho_{\text {grain }}}$

where $\rho_{\text {bulk }}$ is the bulk mass density $\left(\mathrm{ML}^{-3}\right)$ and $\rho_{\text {grain }}$ the particle mass density $\left(\mathrm{ML}^{-3}\right)$, while the effective one, considering the saturated medium, was obtained based on the following relationship:

$\phi_{\text {eff }}=1-\left(\frac{\rho_{\text {bulk }}}{\rho_{\text {grain }}}+\frac{V_{w}}{V}\right)$

where $V\left(\mathrm{~L}^{3}\right)$ is the total volume and $V_{w}\left(\mathrm{~L}^{3}\right)$ the portion of the water volume which cannot be drained by gravity (Staub et al. 2009). The values of main representative parameters for each configuration are shown in Table 2. For further details, see the study of Fallico et al. (2020). The porous media in question can be defined as coarse grained, with a prevalent content of sand and a not negligible amount of gravel. Furthermore, it should be specified that the porous media used here can be considered homogeneous as regards the composition, meaning that the (a)

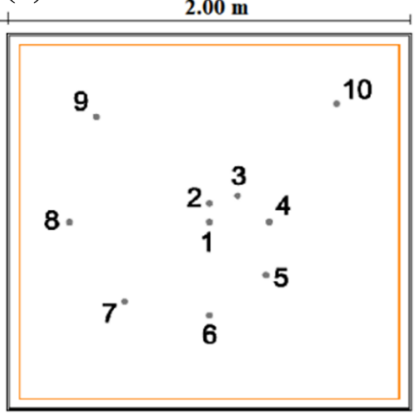

(b)

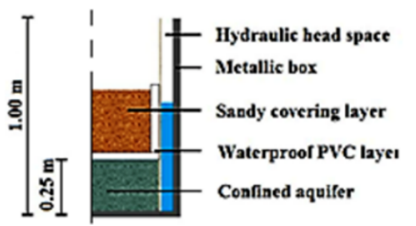

(c)

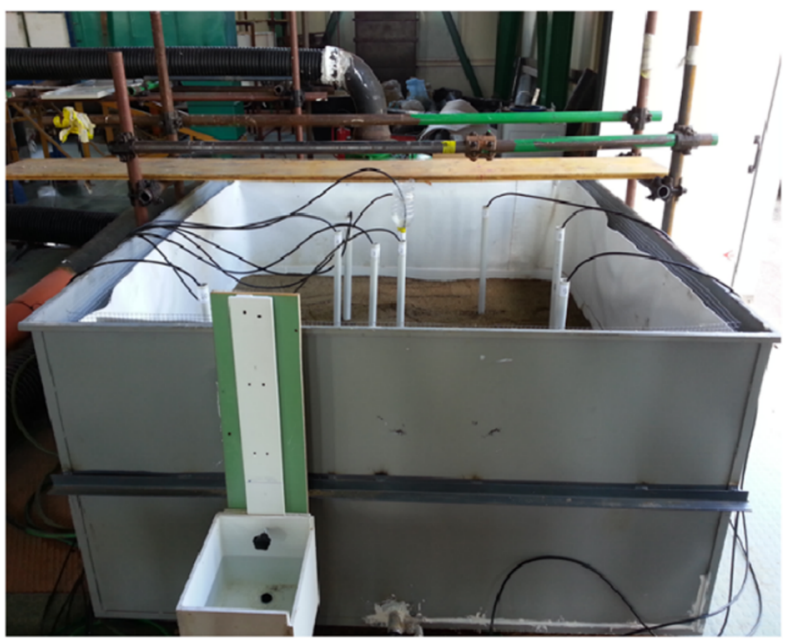


Table 2 Main granulometric and textural parameters of aquifer configurations

\begin{tabular}{lllllllll}
\hline $\begin{array}{l}\text { Porous media } \\
\text { configurations }\end{array}$ & \multicolumn{7}{l}{ Textural parameters and porosity } \\
\cline { 2 - 9 } & $\begin{array}{l}\text { Gravel } \\
(\%)\end{array}$ & $\begin{array}{l}\text { Sand } \\
(\%)\end{array}$ & $\begin{array}{l}\text { Silt } \\
(\%)\end{array}$ & $\begin{array}{l}\text { Clay } \\
(\%)\end{array}$ & $\begin{array}{l}\text { Effective diameter } \\
d_{10}(\mathrm{~mm})\end{array}$ & $\begin{array}{l}\text { Uniformity coefficient } \\
U=d_{60} / d_{10}\end{array}$ & $\begin{array}{l}\text { Total porosity } \\
\phi(\%)\end{array}$ & $\begin{array}{l}\text { Effective } \\
\text { porosity } \phi_{e}(\%)\end{array}$ \\
\hline Type I & 12.01 & 87.39 & 0.60 & - & 0.19 & 5.21 & 37.60 & 5.60 \\
Type II & 27.70 & 71.00 & 1.30 & - & 0.16 & 5.125 & 27.30 & 8.60 \\
Type III & 23.90 & 61.00 & 15.10 & - & 0.02 & 163.5 & 29.30 & 13.00 \\
Type IV & 22.50 & 56.10 & 16.40 & 5.00 & 0.0055 & 163.50 & 19.00 \\
\hline
\end{tabular}

percentages of sand and gravel highlighted above are kept constant in the entire volume of the aquifer considered, while it can certainly be considered heterogeneous in relation to the size, shape and arrangement of the grains.

\section{Methods}

\subsection{Data analysis}

The hydraulic head variations detected during the slug tests were analyzed by the method of Cooper et al. (1967). This is a curve matching method, valid for homogeneous aquifer, unsteady state flow, instantaneous injection and negligible well losses; therefore, careful verification is necessary regarding assumptions on which it is based (Cooper et al. 1967; Butler 1997). For this method the analytical solution can be represented by the following relationship:

$\frac{h(t)}{H_{0}}=f(\beta, \alpha)$

in which $\beta$ represents a dimensionless time parameter:

$\beta=\frac{K B t}{r_{c}^{2}}$

and $\alpha$ represents a dimensionless storage parameter given by:

$\alpha=\frac{r_{w}^{2} S_{s} B}{r_{c}^{2}}$

where $B$ is the aquifer thickness (L), $t$ the time (T), $K$ the radial component of the hydraulic conductivity $\left(\mathrm{LT}^{-1}\right), S_{s}$ the specific storage $\left(\mathrm{L}^{-1}\right), h(t)$ the variation, at time $t$, of the hydraulic head in the well from the initial undisturbed conditions (L), $H_{0}$ the initial value of the hydraulic head in the well (L), $r_{w}$ the effective radius of well screen (L) and $r_{c}$ the effective radius of well casing (L). The radial component of $K$ can be estimated, via curve matching, for $\beta=1$, by the following equation:
$K=\frac{r_{c}^{2}}{B t_{1.0}}$

where $t_{1,0}$ is the real time corresponding to $\beta=1$ (T) and the meaning of the other symbols were already specified (Cooper et al. 1967; Butler 1997).

Before determining the $K$ values, the hydraulic head data series detected in the wells by slug tests were subjected to a careful smoothing analysis, using wavelet transform, specifically the Mexican hat method, to obtain representative data sets and to facilitate data interpretation (Aristodemo et al. 2018).

\subsection{Scaling behavior}

To describe the scaling behavior of some parameters characterizing the aquifer, such as hydraulic conductivity, a power type law is generally used, represented by the following relationship:

$P=a \cdot x^{b}$

where $P$ is the parameter examined (for example the hydraulic conductivity $\left(\mathrm{LT}^{-1}\right)$ ), $x$ is the scale parameter (as distances (L), or corresponding aquifer volume $\left(\mathrm{L}^{3}\right)$ ), $a$ is a parameter linked to the structure and heterogeneity of the medium, with congruence dimensions, and $b(-)$ is the scaling index (or crowding index), which takes into account the type of flow in the porous medium and the actual dimensions of the measurement scale (SchulzeMakuch and Cherkauer 1998). To justify the use of a power law for the description of the scaling behavior, the socalled lacunarity condition must be satisfied, namely it is necessary to identify the variability range of the parameter considered in which the hydrological phenomenon is correctly defined, with the related minimum and maximum cut-off limits (Meakin 1998). Considering the medium in question as a scale-invariant, it is possible to identify the above range and the related cut-off limits with the one that has the maximum value of the determination coefficient $\left(\mathrm{R}^{2}\right)$ (De Bartolo et al. 2013; Fallico et al. 2016, 2018). As regards hydraulic conductivity, the scaling behavior, 
consisting substantially in an increase of $K$ as the scale increases, has been widely investigated (Guimerà et al. 1995; Schulze-Makuch and Cherkauer 1998; Giménez et al. 1999; Schulze-Makuch et al. 1999; Fallico et al. 2012, 2018). Also for porosity, albeit to a lesser extent, the existence of any scaling behavior has been investigated, obtaining, however, results that are not always in agreement (Ewing et al. 2010; Fallico et al. 2010, 2016; Fallico 2014). For other parameters, such as tortuosity, which exerts great influence on the hydraulic conductivity of a porous medium, despite the relevant interest in describing the phenomena of flow and mass transport, aspects related to scaling behavior are poorly investigated. Furthermore, since the radius of influence $(R)$ is often taken as scale parameter, it should be clarified that this represents the characteristic size of the aquifer volume involved in the measurement, of which the value of the measured parameter is representative. In other words, assuming this volume of cylindrical aquifer, as it can be assumed in a homogeneous porous medium in the case of field tests, such as pumping test and slug test, the radius of influence can be assumed equal to the radius of the aforementioned cylindrical volume. By this, it is meant that $R$ is the maximum distance from the pumping or injection well, that is, from the axis of the cylinder, to which it is possible to detect still the stress to which the aquifer is subjected during the test.

\subsection{Description of scaling behavior by fractal analysis}

The models describing the scalar behavior of the above mentioned parameters, in particular of the hydraulic conductivity, are numerous and, obviously, are based on the mutual influences exerted by each of these parameters during the water flow inside the porous medium, described by relationships produced in the various theoretical areas considered.

In all experiments, it resulted

$R_{e}<10$

thus authorizing to regard the flow as laminar. Therefore, the description of the water flow can be performed by the Hagen-Poiseuille law, appropriately applied to the porous media, which, in terms of specific flow rate, assumes the following expression:

$q=\frac{Q}{A}=\frac{\rho g \phi \bar{R}^{2}}{8 \mu \tau_{a v}} \frac{d H}{d x}$

where $q$ is the specific discharge $(\mathrm{L} / \mathrm{T}), A$ the cross-sectional area of the porous medium $\left(\mathrm{L}^{2}\right), \rho$ the water density $\left(\mathrm{ML}^{-3}\right), g$ the gravity acceleration $\left(\mathrm{LT}^{-2}\right), \phi$ the porosity (-), $\mu$ the dynamic viscosity $\left(\mathrm{ML}^{-1} \mathrm{~T}^{-1}\right), \bar{R}$ the average pore radius $(\mathrm{L}), \tau_{a v}$ the average tortuosity $(\mathrm{L}), H$ the hydraulic head (L), $x$ the distance (L) measured in the opposite direction to that of water flow. The definition of tortuosity is not univocal. Here it is assumed for tortuosity the ratio of the effective length of tortuous streamtubes $\left(l_{a}\right)$ and the straight path length $(l)$ in the direction of flow, namely:

$\tau=\frac{l_{a}}{l}$

which, therefore, is always greater than or equal to 1 . In the Eq. (9) the constant of proportionality represents the hydraulic conductivity of the porous medium:

$K=\frac{\rho g \phi \vec{R}^{2}}{8 \mu \tau_{a v}}$

from which it is possible to obtain:

$\tau_{a v}=\frac{\rho g}{\mu} \frac{\phi \bar{R}^{2}}{8 K}$

The generalized Hagen-Poiseuille law relates hydraulic conductivity, identifiable for laminar flow with permeability $(k)$, porosity and tortuosity. The porosity, describes the porous medium structure macroscopically, while the tortuosity describes the microstructure of the medium in which water flow occurs (Dullien 1992; Majumdar 1992; Koponen et al. 1997). Therefore, as evidenced by the relationships (11) and (12), the parameters $K$ and $\tau$ have an evident dependence on porosity. To highlight the functional relationships between the two parameters considered and porosity, as well as experimentally, the fractal approach is increasingly used, which developed from the studies of Jacquin and Adler (1987) and Muller and McCauley (1992) on the representation of the Kozeny (1927) and Carman (1937) equation by the fractal geometry. The model of Muller and McCauley (1992) is base$\mathrm{d}$ on the following relationship:

$K=\phi^{\mu}$

where is called scale crowing index and $\phi$ depends on the measurement scale $(s)$ as well as on the pore size $\lambda$. In this model is taken as a function of the fractal dimension of pores $\left(D_{f}\right)$ and placed equal to:

$\mu=\frac{\left(4-D_{f}\right)}{D_{f}}$

The results provided by this model are very close to those obtainable by Jacquin and Adler's model (1987), which for this reason was not taken into consideration. In this sense, numerous models have been developed (Katz and Thompson 1985; Perrier et al. 1999; Bird and Perrier 2003; $\mathrm{Yu}$ and Cheng 2002). This study is mainly based on the models proposed by $\mathrm{Xu}$ and $\mathrm{Yu}$ (2008) and $\mathrm{Yu}$ and $\mathrm{Li}$ (2004), as well as on the results of a similar experimental 
investigation carried out by De Bartolo et al. (2013) on the scaling behavior of hydraulic conductivity at global scale (laboratory and field).

Established hypotheses of scale invariance and self similarity are valid for the porous medium in question (Korvin 1992; Meakin 1998), in the analytical method used here, porosity and scale were taken as variables to define the parameters in question, by calculating the fractal dimension $\left(D_{f}\right)$ for capillary areas and the fractal dimension $\left(D_{T}\right)$ for tortuous streamtubes in porous media. Given the validity of Eq. (7), this can be determined empirically, assuming the following expression:

$K=a \phi^{b}$

In terms of fractal dimension, the porosity is defined by the following expression ( $\mathrm{Yu}$ and $\mathrm{Li}$ 2001):

$\phi=\left(\frac{\lambda_{\min }}{\lambda_{\max }}\right)^{d_{E}-D_{f}}$

where $\lambda_{\min }$ and $\lambda_{\max }$ are, respectively, the minimum and maximum diameters of the pores, with $\lambda_{\min } \ll \lambda_{\max }, d_{E}$ is the Euclidean dimension ( 2 or 3 ) and $D_{f}$ the fractal dimension of the specific soil type, that is of the specific grain size distribution. According to the model of $\mathrm{Xu}$ and $\mathrm{Yu}$ (2008), the fractal dimension can be explained by the following relationship:

$D_{f}=d_{E}-\frac{\ln \phi}{\ln \left(\frac{\lambda_{\min }}{\lambda_{\max }}\right)}$

and also:

$\lambda_{\max }=d \sqrt{\frac{\phi}{1-\phi}}$

where $d$ is the representative size of the particle, commonly placed equal to $d_{10}$.

Furthermore, again according to the model of $\mathrm{Xu}$ and $\mathrm{Yu}$ (2008), it is possible to define the tortuosity dimension, $D_{\tau}$, by the following relationship:

$D_{\tau}=1+\frac{\ln \tau_{a v}}{\ln \frac{l_{a}}{\lambda_{a v}}}$

where the average tortuosity can be described by the following relation: $\tau_{a v}=\frac{1}{2}\left[1+\frac{1}{2} \sqrt{1-\phi}+\sqrt{1-\phi} \frac{\sqrt{\left(\frac{1}{\sqrt{1-\phi}}-1\right)^{2}+\frac{1}{4}}}{1-\sqrt{1-\phi}}\right]$

while the ratio $\frac{l_{a}}{\lambda_{a v}}$, on the base of geometrical considerations, can be described by the following relationship:

$\frac{l_{a}}{\lambda_{a v}}=\frac{D_{f}-1}{D_{f}^{1 / 2}}\left[\frac{1-\phi}{\phi} \frac{\pi}{4\left(2-D_{f}\right)}\right]^{1 / 2} \frac{\lambda_{\max }}{\lambda_{\min }}$

where according to $\mathrm{Yu}$ and $\mathrm{Li}$ (2001), it is possible to assume:

$\lambda_{a v}=\frac{D_{f}}{D_{f}-1} \lambda_{\min }$

Therefore, the model of Xu and $\mathrm{Yu}$ (2008) describes the relationship between hydraulic conductivity and porosity by the following relationship:

$K=C_{f}\left(\frac{\phi}{1-\phi}\right)^{\left(1+D_{\tau}\right) / 2} \lambda_{\max }^{2}$

where $C_{f}$ is equal to:

$C_{f}=\frac{\left(\pi D_{f}\right)^{\left(1-D_{\tau}\right) / 2}\left[4\left(2-D_{f}\right)\right]^{\left(1-D_{\tau}\right) / 2}}{128\left(3+D_{\tau}-D_{f}\right)}$

The fundamental role of pore size $(\lambda)$ is evident in the above relationships, due to the implicit dependence of the porosity from $\lambda$, but the measurement scale considered is also of great importance, namely the following functional link exists:

$\phi=\phi($ scale,$\lambda)$

However, the determination of $\lambda_{\min }$ and $\lambda_{\max }$ is not easy. $\mathrm{Yu}$ (2005) proposes a method for their determination, which, however, remains quite complex and not without uncertainties.

\section{Results and discussion}

The hydraulic conductivity $(K)$ values were determined for each injection volume and for each configuration taken into consideration. Moreover, for each slug test the corresponding value of the radius of influence $(R)$ was identified and, taking this as scale parameter, the scaling behavior of $K$ was verified, identifying for each configuration the corresponding scaling law, according to the model of power type expressed by the relation (7). The values of $K, R$ and parameters $a$ and $b$ of the scaling laws, obtained according 
to the relation (15), are reported in a previous study of Fallico et al. (2020), to which reference is made for brevity. The scaling behavior of the actual porosity was also verified for each injection volume, taking the radius of influence as scale parameter and identifying the corresponding scaling laws. Afterwards, taking the actual porosity as scale parameter, the scaling laws of $K$ relating to each input volume were identified. The values of the parameters $a$ and $b$ of these scaling laws were obtained, on the basis of relation (15), according to what is set out in the study of Fallico et al. (2020), to which reference is made for further information, and the corresponding values are as follows:

$a=0.0000246 ; \quad b=1.29$

This experimental scaling law is valid for the entire investigation range, relating to all four porous medium configurations considered and, therefore, representative of all coarse grained porous media. This relation, the only one that defines the scalar behavior of $K$ in the whole investigation range in the absence of cut-off limits, defines a simple-scaling behavior of $K$ towards $\phi$. This allows to extend to the whole aquifer in question the self-similarity properties, making possible the use of fractal models for testing.

To describe the variability of $K$ with $\phi$ using the model of $\mathrm{Xu}$ and $\mathrm{Yu}$ (2008) according to the relation (23), it is necessary to keep in mind that $K$ varies also with the fractal dimension of pores $\left(D_{f}\right)$ and with that of tortuosity $\left(D_{\tau}\right)$, respectively according to the relationships (17), (19), (20) and (24). Furthermore, it must be considered that porosity and fractal dimension are highly dependent on the minimum and maximum values of the pore diameters characterizing the saturated porous medium, which are not easy to determine ( $\mathrm{Yu}$ and $\mathrm{Li}$ 2001).

However, as highlighted in the relationships (16), (17), (19) (21), $\lambda_{\min }$ and $\lambda_{\max }$ are always considered in constant ratio for the porous medium under examination, so in the present study the values of the ratio $\lambda_{\min } / \lambda_{\max }$ equal to 0.01 , 0.03 and 0.05 were taken into account. Therefore, the method of $\mathrm{Xu}$ and $\mathrm{Yu}$ (2008) allows to determine on the basis of geometric considerations both $\lambda_{\max }$, by means of the relation (18), and the ratio $\frac{l_{a}}{\lambda_{a}}$, according to the relation (21), bearing in mind that both relations (18) and (21) are functions only of the fractal dimension. In other words, this method allows to assign the upper and lower limits of selfsimilarity in the considered scale, which are of fundamental importance, considering that porosity is a function only of the scale $(s)$ and the size of the pores $(\lambda)$, namely $\phi$ $=\phi(s, \lambda)$.

This consideration highlights the importance of the relationship (18) which provides the value of $\lambda_{\max }$ on which the method of $\mathrm{Xu}$ and $\mathrm{Yu}$ (2008) is substantially based.
Therefore, it emerges that the value of $\lambda_{\max }$ depends strongly on the porosity range taken into consideration from time to time. The trends of $\lambda_{\max } \mathrm{vs} \phi$, for the porous media used in the four configurations under consideration, were shown in Fig. 2, after checking that the values of $d_{10}$, assumed for each configuration of porous medium considered, are acceptable according to (18) and the following relationship, as reported in De Bartolo et al. (2013):

$\int_{0}^{1} \lambda d \phi=\frac{d_{10} \pi}{2}$

The same graph shows the trend observed by De Bartolo et al. (2013), for a porous medium consisting of sandyloam. Figure 2 highlights that the law $\lambda_{\max }=\lambda_{\max }(\phi)$ of the study by De Bartolo et al. (2013), relating to a global scale (laboratory and field), is included between the curves that represent the trend of the law in question for the coarse grained porous media considered here.

Assuming the Euclidean dimension $\left(d_{E}\right)$ equal to 2, determining the values of $D_{f}$ on the basis of the relationship (17), the trend of the fractal dimension of the pores $\left(D_{f}\right)$ as the porosity $(\phi)$ changes, for the above values of the ratio $\lambda_{\min } / \lambda_{\max }$.

Figure 3 highlights that as porosity increases, the influence of the ratio $\lambda_{\min } / \lambda_{\max }$, namely the size of the pores, tends to decrease. The relation (21) allows to determine the ratio $\frac{l_{a}}{\lambda_{a v}}$ as a function of $\phi, D_{f}$ and of the ratio $\lambda_{\min } / \lambda_{\max }$. Therefore, Fig. 4 shows the curves that describe the trend of $\frac{l_{a}}{\lambda_{a v}}$ as the porosity changes $\phi$ for the assigned values of the ratio $\lambda_{\min } / \lambda_{\max }$, taking into account the relationship between porosity and fractal size $D_{f}$. Similarly, Fig. 5 shows the trend of $\frac{l_{a}}{\lambda_{a v}}$ as $D_{f}$ varies, taking into account the relationship $D_{f}=D_{f}(\phi)$.

Since the fractal dimension of the tortuosity $\left(D_{\tau}\right)$ is a function of both the average tortuosity $\tau_{a v}$ and the ratio $\frac{l_{a}}{\lambda_{a v}}$, it is also necessary to determine the value of $\tau_{a v}$ by means of the relation (20), which expresses the exclusive dependence of $\tau_{a v}$ with porosity $(\phi)$. Figure 6 highlights the trend of average tortuosity $\left(\tau_{a v}\right)$ with porosity $(\phi)$. Once the parameters $\tau_{a v}$ and $\frac{l_{a}}{\lambda_{a v}}$ were defined, it is possible to determine the fractal dimension of the tortuosity $\left(D_{\tau}\right)$, by means of the relation (19).

Since both parameters $\tau_{a v}$ and $\frac{l_{a}}{\lambda_{a v}}$ are functions of porosity, consequently also $D_{\tau}$ will result as a function of $\phi$. Figure 7 highlights the trend of $D_{\tau}$ as the porosity changes for the values of the ratio $\lambda_{\min } / \lambda_{\max }$ equal to $0.01,0.03$ and 0.05 respectively.

From the graphs in Figs. 3 and 7, it is possible to note that the trend of the fractal dimension of the pores is opposite to that of the fractal dimension of the tortuosity, 
Fig. 2 Trend of $\lambda_{\max }$ with variations of $\phi$ for the four configurations of porous medium considered and for the porous medium considered in the field investigation by De Bartolo et al. (2013)

Fig. 3 Relationship between fractal dimensions and porosity for different ratios $\lambda_{\min } / \lambda_{\max }$

Fig. 4 Trend of $\frac{l_{a}}{\lambda_{a v}}$ to vary of $\phi$ taking into account the variation of $\phi$ with $D_{f}$, for the fixed values of the ratio $\lambda_{\min } / \lambda_{\max }$ equal to $0.01,0.03,0.05$
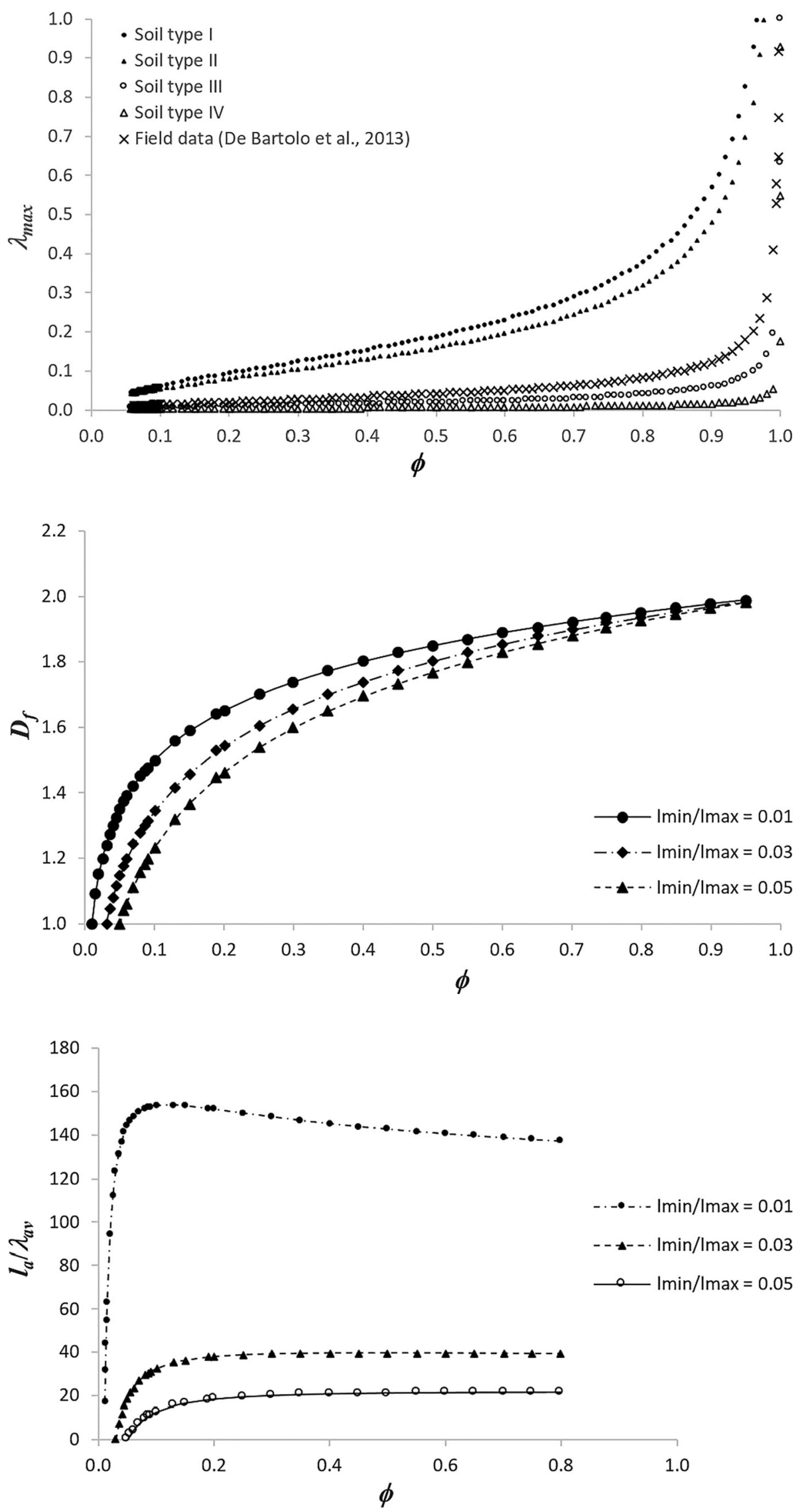
Fig. 5 Trend of $\frac{l_{a}}{\lambda_{a v}}$ to vary of $D_{f}$ taking into account the variation of $D_{f}$ with $\phi$, for the fixed values of the ratio $\lambda_{\text {min }} / \lambda_{\max }$ equal to $0.01,0.03,0.05$

Fig. 6 Trend of average tortuosity $\left(\tau_{a v}\right)$ with porosity $(\phi)$

Fig. 7 Trend of $D_{\tau}$ with variation of $\phi$, for values of the ratio $\lambda_{\text {min }} / \lambda_{\text {max }}$ equal to 0.01 , $0.03,0.05$
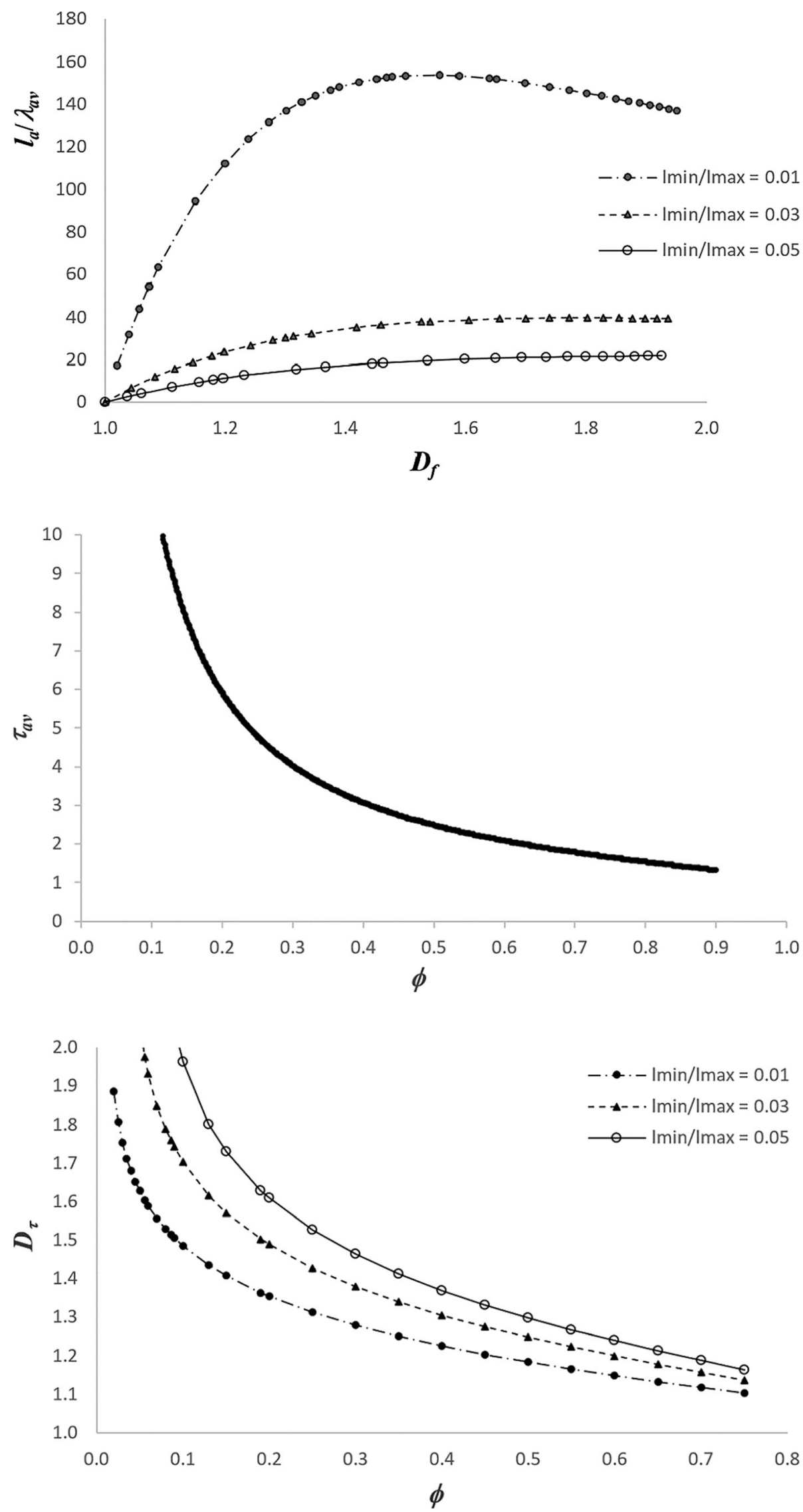
that is an increase in porosity corresponds to an increase in the fractal size $D_{f}$ and a corresponding decrease in the fractal dimension $D_{\tau}$.

This circumstance is better highlighted, for example, by Fig. 8, for the value of the ratio $\lambda_{\min } / \lambda_{\max }$ equal to 0.01 . Finally, to define the variability of the hydraulic conductivity as the porosity changes according to the relationship (23), it is necessary that the parameters $D_{\tau}, \lambda_{\max }$ and the coefficient $\mathrm{C}_{f}$ are known.

The first two parameters $D_{\tau}$ and $\lambda_{\max }$ also depend on $\phi$ and this functional dependence was already highlighted. The coefficient $C_{f}$, defined by the relation (24), is a function of the fractal dimensions $D_{f}$ and $D_{\tau}$, and, since these are functions of $\phi$, the coefficient $C_{f}$ can be also considered dependent on porosity. Therefore the variation modalities of $C_{f}$ with variation of $\phi$ were investigated and the corresponding trend is shown in Fig. 9, for values of $\lambda_{\text {min }} / \lambda_{\text {max }}$ equal to $0.01,0.03,0.05$. The graph in Fig. 9 shows that as $\phi$ increases, the influence of the variation of the ratio $\lambda_{\text {min }} /$ $\lambda_{\text {max }}$ on the value of $C_{f}$ decreases, so that this coefficient tends to a unique constant value. From Eq. (23) it is possible to determine the value of the hydraulic conductivity as the porosity changes, knowing $C_{f}, D_{\tau}$ and $\lambda_{\text {max }}$.

Figure 10 highlights the trend of $K$ with the variation of $\phi$, relative to the fractal model of $\mathrm{Xu}$ and $\mathrm{Yu}$ (2008), for values of $\lambda_{\text {min }} / \lambda_{\text {max }}$ equal to $0.01,0.03,0.05$.

The graph of this figure shows also the experimental law obtained from Fallico et al. (2020), described by the relationship (26), and the law described by the relationship (13), according to the model of Muller and McCauley (1992), for values of $\lambda_{\min } / \lambda_{\max }$ equal to $0.01,0.03,0.05$.

The trends described show a clear increase of $K$ with an increase of $\phi$. The model of $\mathrm{Xu}$ and $\mathrm{Yu}$ (2008) provides almost coincident trends, as the ratio $\lambda_{\text {min }} / \lambda_{\text {max }}$ changes, and this proximity tends to increase with an increase of $\phi$.
Furthermore, the performance of $K$ relating to this model is almost coincident with that of the experimental law of the relationship (13). Only in the final part, for values of $\phi$ greater than $35-40 \%$, the trend of the experimental law differs from that of the $\mathrm{Xu}$ and Yu model (2008), providing lower values of $K$ than those obtainable with this fractal model. The fractal model of Muller and McCauley (1992) gives significantly higher values of $K$ than those obtained both with the experimental law (13) and with the fractal model of $\mathrm{Xu}$ and $\mathrm{Yu}$ (2008). Furthermore, with this fractal model, as the $\lambda_{\text {min }} / \lambda_{\text {max }}$ ratio changes, the $K$ values diverge for low values of $\phi$, while they tend to coincide for increasing porosity values. The graph of Fig. 10 shows also that as regards the relationship (13) of Muller and McCauley (1992) in a first range, for $\phi$ between 0.056 and about 0.21 , it can clearly foresee a multi-scaling behavior of $K$, as the ratio $\lambda_{\min } / \lambda_{\max }$ varies. On the contrary, as regards the relationship (23) of $\mathrm{Xu}$ and $\mathrm{Yu}$ (2008), the same graph of Fig. 10 shows that there is a first range with evident simple-scaling behavior, which runs from values of $\phi$ equal to 0.056 up to about 0.28 , and then a second range with a different trend, showing an increase of $K$ for the remaining part. This latter behavior is in accordance with what was found by Severino and De Bartolo (2019) in a recent study concerning the water retention curves.

Table 3 shows the parameters that characterize mostly the models taken into consideration here and that highlight the proximity of the model of $\mathrm{Xu}$ and $\mathrm{Yu}$ (2008), represented by the relation (23), to the experimental law represented by the relation (26) and the scarce representativeness of the Muller and McCauley model (1992), represented by the relationships (13) and (14).

The data in Table 3, in particular the RMSE values, confirms what is highlighted in Fig. 10, namely that the $\mathrm{Xu}$ and $\mathrm{Yu}$ (2008) model approximates the experimental law
Fig. 8 Opposite trends of the pore area fractal dimension and tortuosity fractal dimension with porosity, for $\lambda_{\min } / \lambda_{\max }$ equal to 0.01

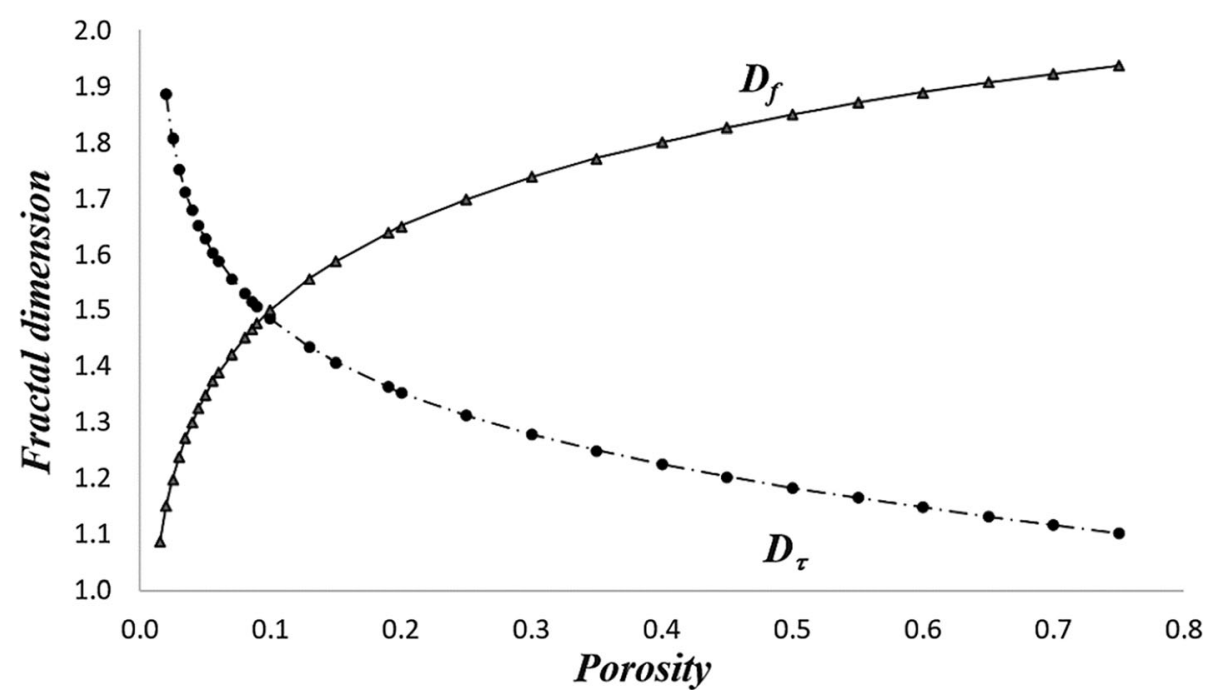


Fig. 9 Trend of the coefficient $C_{f}$ with variation of $\phi$, for values of the ratio $\lambda_{\min } / \lambda_{\max }$ equal to $0.01,0.03,0.05$
Fig. 10 Trend of $K$ with variations of $\phi$ according to the fractal models of $\mathrm{Xu}$ and $\mathrm{Yu}$ (2008) and Muller and McCauley (1992), for values of $\lambda_{\text {min }} / \lambda_{\text {max }}$ equal to $0.01,0.03$, 0.05 , and according to the experimental law given from the relationship (13) (Fallico et al. 2020)
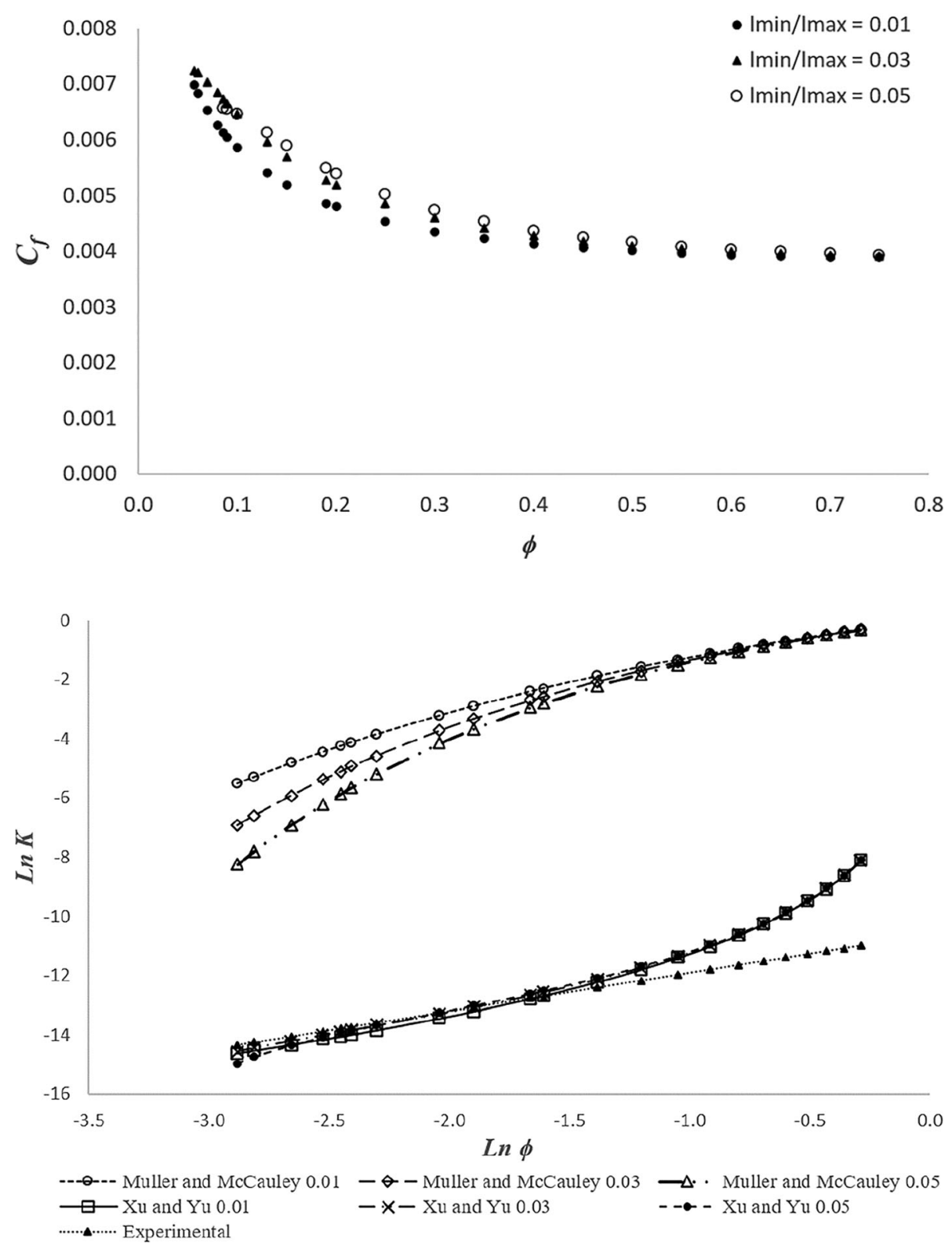

well, certainly in a much better way than the Muller and McCauley model (1992). Also in the study of De Bartolo et al. (2013) the Muller and McCauley model (1992) interprets the experimental model less well than the $\mathrm{Xu}$ and Yu model (2008), with higher RMSE values. However, the difference was not as sharp as in the case examined here. Since the values of the porosity and the dimensions of the voids, for the porous media considered in the two investigations, can be considered comparable, a probable hypothesis to explain the clear difference between the interpretation of the experimental law provided by the Muller and McCauley model (1992) in the study of De Bartolo et al. (2013) and that relating to this investigation could be found in the different investigation scale considered in the two studies. In fact, in the study by De Bartolo et al. (2013) was considered a global scale, in particular laboratory and open field, while in the present study an intermediate scale between the laboratory and the field was considered.

Furthermore, it should be noted that the comparison between the graph of Fig. 6 and that of Fig. 10 shows opposite trends of $K$ and $\tau_{a v}$, with variation of $\phi$. These trends agree with what was stated in the relationships (11) and (12) and an increase in $K$ corresponds to a decrease in $\tau_{a v}$

In fact, when $\tau_{a v}$ decreases, the resistance to water flow within the canaliculi, namely the pressure drop, decreases, and consequently $K$ increases, as the porous medium becomes more conductive. The opposite happens when $\tau_{a v}$ increases. 
Table 3 Values of the main characteristic parameters of the fractal models considered and of the experimental one, relative to the four configurations of porous medium considered and for values of $\lambda_{\text {min }} / \lambda_{\text {max }}$ equal to $0.01,0.03$ and 0.05

\begin{tabular}{|c|c|c|c|c|c|c|}
\hline \multicolumn{3}{|l|}{ Models } & Configuration type & $\mu$ & $D_{f}$ & RMSE \\
\hline \multirow{4}{*}{\multicolumn{3}{|c|}{ Experimental law }} & I & 1.29 & - & $4.33 \times 10^{-5}$ \\
\hline & & & II & & & $1.60 \times 10^{-4}$ \\
\hline & & & III & & & $7.29 \times 10^{-5}$ \\
\hline & & & IV & & & $1.78 \times 10^{-4}$ \\
\hline \multirow[t]{12}{*}{ Muller and McCauley (1992) } & \multirow[t]{12}{*}{$\lambda_{\min } / \lambda_{\max }$} & \multirow[t]{4}{*}{0.01} & I & 1.911 & 1.374 & \multirow[t]{4}{*}{$3.43 \times 10^{-1}$} \\
\hline & & & II & 1.726 & 1.467 & \\
\hline & & & III & 1.569 & 1.557 & \\
\hline & & & IV & 1.440 & 1.639 & \\
\hline & & \multirow[t]{4}{*}{0.03} & $\mathrm{I}$ & 2.396 & 1.178 & \multirow[t]{4}{*}{$3.33 \times 10^{-1}$} \\
\hline & & & II & 2.076 & 1.300 & \\
\hline & & & III & 1.820 & 1.418 & \\
\hline & & & IV & 1.620 & 1.526 & \\
\hline & & \multirow[t]{4}{*}{0.05} & I & 2.854 & 1.038 & \multirow[t]{4}{*}{$3.27 \times 10^{-1}$} \\
\hline & & & II & 2.387 & 1.181 & \\
\hline & & & III & 2.033 & 1.319 & \\
\hline & & & IV & 1.767 & 1.446 & \\
\hline \multirow[t]{12}{*}{$\mathrm{Xu}$ and Yu (2008) } & \multirow[t]{12}{*}{$\lambda_{\min } / \lambda_{\max }$} & \multirow[t]{4}{*}{0.01} & I & - & 1.374 & \multirow[t]{4}{*}{$7.59 \times 10^{-5}$} \\
\hline & & & II & & 1.467 & \\
\hline & & & III & & 1.557 & \\
\hline & & & IV & & 1.639 & \\
\hline & & \multirow[t]{4}{*}{0.03} & I & & 1.178 & \multirow[t]{4}{*}{$7.60 \times 10^{-5}$} \\
\hline & & & II & & 1.300 & \\
\hline & & & III & & 1.418 & \\
\hline & & & IV & & 1.526 & \\
\hline & & \multirow[t]{4}{*}{0.05} & $\mathrm{I}$ & & 1.038 & \multirow[t]{4}{*}{$7.61 \times 10^{-5}$} \\
\hline & & & II & & 1.181 & \\
\hline & & & III & & 1.319 & \\
\hline & & & IV & & 1.446 & \\
\hline
\end{tabular}

\section{Conclusions}

The present study shows the results of an investigation carried out on confined aquifers, built in a special laboratory device using four different configurations of porous media, which can be assumed to be sufficiently representative of the coarse grained media and which, as explained above, can be considered homogeneous. For each of these configurations a careful analysis of direct scaling for both hydraulic conductivity and porosity was performed, taking into account that all the measurements carried out refer to the particular investigation scale, which is intermediate between the proper laboratory scale (samples of porous media) and that characteristic of field. This analysis was carried out considering only simple-scaling behavior for the parameters taken into consideration, excluding for the sake of simplicity any multi-scaling behaviors. The scaling analysis was carried out on the basis of a power type law, which allowed the use of fractal models. In fact, in this way it was possible to express the scale index of the power law as a function of the fractal dimension and, therefore, to determine the value of this parameter. Therefore, it was possible to make comparisons between the law $K=K(\phi)$, obtained experimentally, and those expressed through the use of known fractal models, in particular that of $\mathrm{Xu}$ and $\mathrm{Yu}$ (2008) and that of Muller and McCauley (1992).

The analysis of the results obtained made it possible to estimate the level of uncertainty of the different models used in terms of RMSE, and to carry out the appropriate comparisons. The results obtained showed that the model of $\mathrm{Xu}$ and $\mathrm{Yu}$ (2008) is more reliable than that of Muller and McCauley (1992), managing to interpret the law (26), obtained experimentally, with high degree of reliability. This result is confirmed by the values of the root mean square error obtained for the models taken into consideration (see Table 3). In fact, the RMSE values relating to the model of $\mathrm{Xu}$ and $\mathrm{Yu}(2008)$ are the lowest and of the same order of magnitude as those of the experimental law, while that relating to the model of Muller and McCauley (1992) is considerably higher. From the comparison with a 
previous study (De Bartolo et al. 2013), in which the Muller and McCauley model (1992) provided a representation of the scaling law closer to the experimental one, albeit with less reliability than the $\mathrm{Xu}$ and $\mathrm{Yu}$ (2008), the influence of the particular mesoscale taken into consideration emerges. This represents only a limited part, intermediate between the laboratory and field scale, of the general scale (laboratory and field) considered in the study by De Bartolo et al. (2013). This seems to confirm the peculiarities of this mesoscale, already highlighted in previous studies (Fallico et al. 2018, 2020).

Uncertainty quantification attached to the use of single models allows a more reliable description of flow and transport phenomena. The present study goes along such an avenue, and in particular it provides insights on important environmental topics, such as protection and management of water resources.

Funding Open access funding provided by Università degli Studi di Napoli Federico II within the CRUI-CARE Agreement.

Open Access This article is licensed under a Creative Commons Attribution 4.0 International License, which permits use, sharing, adaptation, distribution and reproduction in any medium or format, as long as you give appropriate credit to the original author(s) and the source, provide a link to the Creative Commons licence, and indicate if changes were made. The images or other third party material in this article are included in the article's Creative Commons licence, unless indicated otherwise in a credit line to the material. If material is not included in the article's Creative Commons licence and your intended use is not permitted by statutory regulation or exceeds the permitted use, you will need to obtain permission directly from the copyright holder. To view a copy of this licence, visit http://creativecommons. org/licenses/by/4.0/.

\section{References}

Agboola O, Onyango MS, Popoola P, Oyewo OA (2017) Fractal geometry and porosity. In: Fractal analysis: applications in physics, engineering and technology, Chapter 10, published by Intech. https://doi.org/10.5772/intechopen.68201

Ahuja LR, Cassel DK, Bruce RR, Barnes BB (1989) Evaluation of spatial distribution of hydraulic conductivity using effective porosity data. Soil Sci 148(6):404-411

Aristodemo F, Ianchello M, Fallico C (2018) Smoothing analysis of slug tests data for aquifer characterization at laboratory scale. J Hydrol 562:125-139

Bird N, Perrier E (2003) The pore-solid fractal model of soil density scaling. Eur J Soil Sci 54:467-476

Bouma J (1982) Measuring the hydraulic conductivity of soil horizons with continuous macropores. Soil Sci Soc Am J 46(2):438-441

Butler JJ Jr (1997) The design, performance, and analysis of slug tests. Lewis Publishers, Boca Raton

Carman PC (1937) Fluid flow through granular beds. Trans Inst Chem Eng 15:150-166

Chen X, Yao G (2017) An improved model for permeability estimation in low permeable porous media based on fractal geometry and modified Hagen-Poiseuille flow. Fuel 210(15):748-757

Chen H, Yang M, Chen K, Zhang C (2020) Relative permeability of porous media with nonuniform pores. Geofluids. https://doi.org/ $10.1155 / 2020 / 5705424$

Clauser C (1992) Permeability of crystalline rocks. EOS Trans Am Geophys Union 73(21):233-238

Comegna A, Coppola A, Comegna V, Severino G, Sommella A, Vitale CD (2010) State-space approach to evaluate spatial variability of field measured soil water status along a line transect in a volcanic-vesuvian soil. Hydrol Earth Syst Sci $14: 2455-2463$

Cooper HH, Bredehoeft JD, Papadopulos IS (1967) Response of a finite-diameter well to an instantaneous charge of water. Water Resour Res 3:263

Danielson RE, Sutherland PL (1986) Methods of soil analysis-part 1. Physical and mineralogical methods; agronomy monograph; Soil Science Society of America: Madison, WI, USA, vol 9, pp 443-461

De Bartolo S, Fallico C, Veltri M (2013) A note on the fractal behavior of hydraulic conductivity and effective porosity for experimental values in a confined aquifer. Sci World $\mathbf{J}$ 2013:1-10

Di Federico V, Neuman SP, Tartakovsky DM (1999) Anisotropy, lacunarity, upscaled conductivity, and its covariance in multiscale random fields with truncated power variograms. Water Resour Res 35(10):2891-2908

Dullien FAL (1992) Porous media: fluid transport and pore structure, 2nd edn. Academic Press, San Diego, p 574

Ewing RP, Hu Q, Liu C (2010) Scale dependence of intragranular porosity, diffusivity, and tortuosity. Water Resour Res 46:W06513. https://doi.org/10.1029/2009WR008183

Fallico C (2014) Reconsideration at Field Scale of the Relationship between hydraulic conductivity and porosity. The case of a sandy aquifer in South Italy. Sci World J Article ID 537387, 15 p. https://doi.org/10.1155/2014/537387

Fallico C, De Bartolo S, Troisi S, Veltri M (2010) Scaling analysis of hydraulic conductivity and porosity on a sandy medium of an unconfined aquifer reproduced in the laboratory. Geoderma 160(1):3-12

Fallico C, Vita MC, De Bartolo S, Straface S (2012) Scaling effect of the hydraulic conductivity in a confined aquifer. Soil Sci 177(6):385-391

Fallico C, De Bartolo S, Veltri M, Severino G (2016) On the dependence of the saturated hydraulic conductivity upon the effective porosity through a power law model at different scales. Hydrol Process 30:2366-2372. https://doi.org/10.1002/hyp. 10798

Fallico C, Ianchello M, DeBartolo S, Severino G (2018) Spatial dependence of the hydraulic conductivity in a well-type configuration at the mesoscale. Hydrol Process 32(4):590-595. https:// doi.org/10.1002/hyp.11422

Fallico C, Lauria A, Aristodemo F (2020) Porous medium typology influence on the scaling laws of confined aquifer characteristic parameters. Water 12:1166. https://doi.org/10.3390/w12041166

Franzmeier DP (1991) Estimation of hydraulic conductivity from effective porosity data for some Indiana soils. Soil Sci Soc Am J 55(6): 1801-1803

Ghanbarian B, Hunt AG, Ewing RP, Sahimi M (2012) Tortuosity in porous media: a critical review. Soil Sci Soc Am J 77:1461-1477. https://doi.org/10.2136/sssaj2012.0435

Giménez D, Rawls WJ, Lauren JG (1999) Scaling properties of saturated hydraulic conductivity in soil. Geoderma 88(3-4):205-220 
Guimerà J, Vives L, Carrera J (1995) A discussion of scale effects on hydraulic conductivity at a granitic site (El Berrocal, Spain). Geophys Res Lett 22(11):1449-1452

Hyun Y, Neuman SP, Vesselinov VV, Illman WA, Tartakovsky DM, Di Federico V (2002) Theoretical interpretation of a pronounced permeability scale effect in unsaturated fractured tuff. Water Resour Res 38(6): 1092

Jacquin CG, Adler PM (1987) Fractal porous media II: geometry of porous geological structures. Transp Porous Med 2(6):571-596

Katz AJ, Thompson AH (1985) Fractal sandstone pores: implications for conductivity and pore formation. Phys Rev Lett 54(12): 1325-1328

Knudby C, Carrera J (2006) On the use of apparent hydraulic diffusivity as an indicator of connectivity. J Hydrol 329(3-4):377-389

Koponen A, Kataja M, Timonen J (1997) Permeability and effective porosity of porous media. Phys Rev E 56:3319

Korvin G (1992) Fractal models in the earth sciences. Elsevier Science, Oxford

Kozeny J (1927) Uber kapillare Leitung des Wassers in Boden. Sitzungsberichte dr Wiener Akademie des Wissenschaften 2:136-306 (German)

Lambe TW (1951) Soil testing for engineers. Wiley, New York

Majumdar A (1992) Role of fractal geometry in the study of thermal phenomena. Ann Rev Heat Transf, IV, pp 51-110

Meakin P (1998) Fractals, scaling and growth far from equilibrium. Cambridge University Press, Cambridge

Muller J, McCauley JL (1992) Implication of fractal geometry for fluid flow properties of sedimentary rocks. Transp Porous Med $8(2): 133-147$

Perrier E, Bird N, Rieu M (1999) Generalizing the fractal model of soil structure: the PSF approach. Geoderma 88:137-164

Rovey CW II, Cherkauer DS (1994) Relation between hydraulic conductivity and texture in a carbonate aquifer: observations. GroundWater 32(1):53-62

Schulze-Makuch D, Cherkauer DS (1997) Method developed for extrapolating scale behavior. EOS Trans Am Geophys Union 78(13):3-7

Schulze-Makuch D, Cherkauer DS (1998) Variations in hydraulic conductivity with scale of measurement during aquifer tests in heterogeneous, porous carbonate rocks. Hydrogeol J 6(2):204-215

Schulze-Makuch D, Carlson DA, Cherkauer DS, Malik P (1999) Scale dependency of hydraulic conductivity in heterogeneousmedia. GroundWater 37(6):904-919

Severino G (2011a) Macrodispersion by source flows in randomly heterogeneous porous media. Transp Porous Media 89:121-134

Severino G (2011b) Stochastic analysis of well-type flows in randomly heterogeneous porous formations. Water Resour Res 47:W03520. https://doi.org/10.1029/2010WR009840

Severino G, Coppola A (2012) A note on the apparent conductivity of stratified porous media in unsaturated steady flow above a water table. Transp Porous Med 91(2):733-740

Severino G, De Bartolo S (2019) A scale invariant property of the water retention curve in weakly heterogeneous vadose zone. Hydrol Process 33(7):1032-1039
Severino G, Santini A (2005) On the effective hydraulic conductivity in mean vertical unsaturated steady flows. Adv Water Resour 28:964-974

Severino G, Monetti VM, Santini A, Toraldo G (2006) Unsaturated transport with linear kinetic sorption under unsteady vertical flows. Transp Porous Med 63:147-174

Severino G, Santini A, Monetti VM (2009) Modelling water flow and solute transport in heterogeneous porous media. In: Papajorgji PJ, Pardalos PM (eds) Advances in modeling agricultural systems. Springer, New York, pp 361-383

Severino G, Comegna A, Coppola A, Sommella A, Santini A (2010) Stochastic analysis of a field-scale unsaturated transport experiment. Adv Water Resour 33:1188-1198

Severino G, Santini A, Sommella A (2011) Macrodispersion by diverging radial flows in randomly heterogeneous porous media. J Contam Hydrol 123:40-49

Severino G, Tartakovsky D, Srinivasan G, Viswanathan H (2012) Lagrangian models of reactive transport in heterogeneous porous media with uncertain properties. Proceedings of the Royal Society A 468:1154-1174

Severino G, DeBartolo S, Brunetti G, Sommella A, Fallico C (2019) Experimental evidence of the stochastich behavior of the conductivity in radial flow configurations. Stochas Environ Res Risk Assess 33(8):1651-1657. https://doi.org/10.1007/s00477019-01704-z

Staub M, Galietti B, Oxarango L, Khire MV, Gourc JP (2009) Porosity and hydraulic conductivity of MSW using laboratoryscale tests. In: Proceedings of the 3rd international workshop "hydro-physico-mechanics of landfills", Braunschweig, Germany, 10-13 March

Vidales AM, Miranda EN (1996) Fractal porous media: relations between macroscopic properties. Chaos Solitons Fract 7(9):1369-1996

Wheatcraft SW, Tyler SW (1988) An explanation of scale-dependent dispersivity in heterogeneous aquifers using concepts of fractal geometry. Water Resour Res 24(4):566-578. https://doi.org/10. 1029/WR024i004p00566

Winter CL, Tartakovsky DM (2001) Theoretical foundation for conductivity scaling. Geophys Res Lett 28(23):4367-4370

$\mathrm{Xu}$ P, Yu BM (2008) Developing a new form of permeability and Kozeny-Carman constant for homogeneous porous media by means of fractal geometry. Adv Water Resour 31(1):74-81

Yanuka M, Dullien FAL, Elrick DE (1986) Percolation processes and porous media: I-geometrical and topological model of porous media using a three-dimensional joint pore size distribution. J Colloid Interface Sci 112(1):24-41

Yu BM (2005) Fractal character for tortuous streamtubes in porous media. Chin Phys Lett 22(1):158

Yu BM, Cheng P (2002) A fractal permeability model for bidispersed porousmedia. Int J Heat Mass Transf 45(14):2983-2993

Yu BM, Li J (2001) Some fractal characters of porous media. Fractals 9(3):365-372

Yu BM, Li JH (2004) A geometry model for tortuosity of flow path in porous media. Chin Phys Lett 21(8):1569-1571

Publisher's Note Springer Nature remains neutral with regard to jurisdictional claims in published maps and institutional affiliations. 